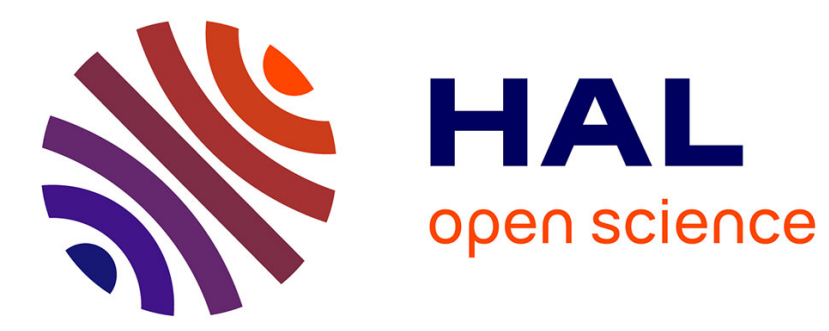

\title{
La réforme de l'intercommunalité
}

Cécile Jebeili

\section{- To cite this version:}

Cécile Jebeili. La réforme de l'intercommunalité. GREP-POUR, 2011, 209-210, pp.49-63. hal00812676

\section{HAL Id: hal-00812676 \\ https://hal-univ-tlse2.archives-ouvertes.fr/hal-00812676}

Submitted on 12 Apr 2013

HAL is a multi-disciplinary open access archive for the deposit and dissemination of scientific research documents, whether they are published or not. The documents may come from teaching and research institutions in France or abroad, or from public or private research centers.
L'archive ouverte pluridisciplinaire HAL, est destinée au dépôt et à la diffusion de documents scientifiques de niveau recherche, publiés ou non, émanant des établissements d'enseignement et de recherche français ou étrangers, des laboratoires publics ou privés. 


\section{LA REFORME DE L'INTERCOMMUNALITE}

La réforme territoriale promulguée le 16 décembre dernier ${ }^{1}$, après plus d'un an de débats parlementaires nécessitant pas moins de trois lectures entre les deux chambres, rate cependant largement les objectifs de simplification et de clarification que le président de la République lui avait assignés. Un volet de la réforme a néanmoins fait l'objet d'un relatif consensus, il s'agit du volet intercommunal qui fait émerger un nouveau degré d'administration locale : « la commune dans sa communauté ».

Ce nouveau couple local, formé au travers d'une plus grande solidarité financière et fiscale, d'une mutualisation de ses services, d'une nouvelle forme de gouvernance démocratique, et dans un cadre géographique rénové, pragmatique et rationnel, saura peut-être inventer de nouvelles formes d'articulations politiques pour créer un territoire favorable au développement d'une citoyenneté intercommunale et pourra sans doute devenir un jour une seule et même collectivité.

\section{Les territoires}

\section{a. Le constat}

Au $1^{\text {er }}$ janvier 2011, $2599 \mathrm{EPCl}$ à FP réunissaient plus de 95,5\% des communes et 89,9\% de la population. Il n'est pas inapproprié de parler de "révolution intercommunale " et c'est essentiellement la loi Chevènement du 12 juillet 1999 qui a permis de réaliser un tel succès après plus de 40 ans de tentatives infructueuses de maillage intercommunal du territoire national.

Jusque-là, le paysage intercommunal français était en effet assez désolé, voire désolant : les formules intercommunales proposées au fil des lois se superposaient sans aucune cohérence, sans qu'on puisse les distinguer vraiment les unes des autres ni les identifier clairement; la large palette des mécanismes fiscaux ne correspondait pas nécessairement à un niveau d'intégration de compétences, lequel n'était d'ailleurs pas nécessairement exercé dans la pratique ; l'exigence de cohérence spatiale des intercommunalités était réduite à sa plus simple expression ${ }^{2}$ et une commune pouvait adhérer à plusieurs $\mathrm{EPCl}^{3}$. Il était d'ailleurs clair que beaucoup EPCl étaient alors des " coquilles vides" ou des intercommunalités dites "d'aubaine " créées pour des raisons strictement financières et que de nombreuses "intercommunalités défensives", au périmètre souvent trop étroit, au faible poids démographique, dotées d'un potentiel financier insuffisant, se constituaient à la périphérie

\footnotetext{
${ }^{1}$ Loi $n^{\circ} 2010-1563$ du 16 décembre 2010 de réforme des collectivités territoriales.

${ }^{2}$ La contiguïté territoriale des communes.

${ }^{3}$ En pratique, c'était d'ailleurs la règle, et en moyenne, une commune adhérait en 1998 à 5,4 groupements, entraînant un enchevêtrement des relations entre communes et in fine, une succession de dysfonctionnements : chevauchement de compétences, délégation d'une même compétence à plusieurs $\mathrm{EPCl}$, exercice par une commune d'une compétence déléguée à un EPCl, exercice par un EPCI d'une compétence qui ne lui a pas été déléguée ...
} 
des villes-centres, fractionnant ainsi les aires urbaines en une mosaïque, voire une « macédoine » de petites intercommunalités.

La réforme Chevènement a donc d'abord et avant tout fait œuvre de simplification et de rationalisation de l'intercommunalité, d'une part en accentuant l'exigence de cohérence territoriale ${ }^{4}$ et en supprimant la possibilité pour une commune de multiplier les regroupements intercommunaux ${ }^{5}$ et $d^{\prime}$ 'autre part, en réduisant l'offre intercommunale à trois principales formules, organisées de façon pyramidale en fonction d'un niveau d'intégration fiscale et fonctionnelle allant de pair avec le poids démographique des communautés. La loi a ainsi construit une architecture structurée, lisible et cohérente autour de la communauté de communes $(C C)^{6}$, de la communauté d'agglomération $(C A)^{7}$ et enfin de la communauté urbaine $(\mathrm{CU})^{8}$.

L'objectif était d'engager les communes dans un processus inéluctable d'intercommunalisation et de remporter le pari du maillage intercommunal total du territoire national à l'horizon 2010.

Or, au $1^{\text {er }}$ janvier 2010, 1908 communes isolées regroupant un petit peu plus de 7 millions d'habitants, soit $5 \%$ des communes françaises et près de $11 \%$ de la population totale constituent encore ces fameuses zones blanches qui résistent encore au « rouleau compresseur $»$ intercommunal ${ }^{9}$.

Par ailleurs, depuis le rapport assez accablant rendu par la Cour des comptes en novembre $2005^{10}$, et réitéré ensuite en $2009^{11}$ on sait en effet qu'il subsiste encore trop d'EPCI à FP trop étroits et/ ou d'un trop faible poids démographique ${ }^{12}$.

Ainsi, au $1^{\text {er }}$ janvier 2010, si la taille moyenne d'une communauté est de 13,3 communes et son poids de 22193 habitants, ce qui apparaît satisfaisant au regard des critères de taille et poids critiques posés par la Cour des comptes ${ }^{13}$, on relève cependant un nombre encore trop importants de cas limites.

\footnotetext{
${ }^{4}$ Nécessité d'un périmètre d'un seul tenant et sans enclave.

${ }^{5}$ Dorénavant, l'article L. 5210-2 CGCT dispose : "Une commune ne peut appartenir à plus d'un EPCI à fiscalité propre", c'est à dire hors syndicats de communes et syndicats mixtes.

${ }^{6}$ Formule la plus souple et la moins intégrée, elle est dédiée aux plus petites communes, elle n'est donc pas soumise à un seuil démographique, elle est dotée de compétences obligatoires limitées et ajustables, et d’une large palette fiscale, allant de la simple fiscalité additionnelle jusqu'à la TPU, en passant par la TPZ.

${ }^{7}$ Cette formule nouvelle introduit pour la première fois dans notre législation la notion d'agglomération, et s'adresse aux aires urbaines de plus de 50000 habitants autour d'une ville-centre de 15000 habitants. C'est une formule très intégrée, aux larges compétences, notamment en termes d'aménagement de l'espace et de développement économique, dotée obligatoirement de la TPU.

${ }^{8}$ Formule la plus intégrée, dont le seuil démographique fixé à 20000 habitants en 1992 a été largement relevé à 500000 habitants pour s'adresser aux grandes métropoles régionales. La communauté urbaine est dotée des compétences les plus étendues et largement incitée à adopter la TPU à compter de 2002.

${ }^{9}$ Au cours de l'année 2010, ce sont 125 communes isolées qui ont adhéré à des regroupements existants.

${ }^{10}$ Cour des comptes, L'intercommunalité en France, Rapport remis au Président de la République, La Documentation Française, 2005.

${ }^{11}$ Cour des comptes, Bilan d'étape de I'Intercommunalité en France in Rapport annuel 2009, La Documentation Française, 2009.

12 V. notre article: L'intercommunalité à la recherche du territoire pertinent, La Semaine Juridique, Administrations et collectivités territoriales, 3 novembre 2008, $n^{\circ} 45$, p. 14 et suiv., v. p. 20 et suiv.

${ }^{13}$ + de 10000 habitants et/ou + de 10 communes : Bilan d'étape de l'Intercommunalité en France, prec.
} 
En ce qui concerne la taille des intercommunalités, le nombre de communautés comptant moins de 5 communes est encore aujourd'hui de $13 \%$, et en ce qui concerne leur poids, la part des communautés de moins de 5000 habitants est d'un peu plus du quart ${ }^{14}$, et il reste encore $11 \mathrm{CC}$ de moins de 700 habitants et 15 comprises entre 700 et 999 habitants.

Si le phénomène remarquable de la fragmentation intercommunale des aires urbaines est essentiellement lié à des stratégies de regroupement qui n'ont évidemment rien à voir avec les objectifs de cohérence et de solidarité territoriale (les fameux "clubs de riches » et les " intercommunalités défensives »), en milieu rural en revanche, la situation est plus nuancée.

Le poids démographique insuffisant de certaines communautés de communes est bien entendu lié à la faible densité de ce milieu ${ }^{15}$, mais également au fait que le canton a beaucoup présidé au découpage des périmètres intercommunaux en milieu rural, parfois parce que le chef-lieu de canton exerce un rôle de centralité pertinent, beaucoup parce que les conseillers généraux se sont "emparés" de l'intercommunalité. Ce "leadership » s'explique bien souvent par le fait que le conseiller général est aussi le maire du chef-lieu de canton, et devient donc naturellement président d'une intercommunalité cantonale, ce qui fait que ces intercommunalités ne se fondent pas nécessairement sur des espaces de solidarité mais sont plutôt l'objet d'une construction politique menée par les pouvoirs locaux en place. Il y a donc aujourd'hui 1235 CC, soit près de la moitié d'entre elles, qui ont encore un périmètre inférieur ou égal au canton.

\section{b. Les dispositions de la loi du $\mathbf{1 6}$ décembre $\mathbf{2 0 1 0}$}

Un chapitre entier de la loi du 16 décembre 2010 est donc consacré à la question de l'achèvement et de la rationalisation de la carte de l'intercommunalité ${ }^{16}$.

La date d'achèvement de la carte de l'intercommunalité est finalement fixée au $1^{\text {er }}$ juin $2013^{17}$ et les Commissions Départementales de la Coopération Intercommunale (CDCI) doivent être renouvelées dans les 3 mois de la promulgation de la loi de façon à renforcer la représentation des intercommunalités en leur $\operatorname{sein}^{18}$. Elles doivent adopter le Schéma Départemental de la Coopération Intercommunale (SDCI) en concertation avec le préfet au plus tard le 31 décembre 2011.

\footnotetext{
${ }^{14} 668 \mathrm{CC}$.

15 Moins de 50 habitants/ $\mathrm{km}^{2}$ pour les «communautés rurales » contre 350 habitants/ km² pour les communautés "urbaines» (Chiffres: ADCF-Observatoire de l'intercommunalité, Portrait des intercommunalités rurales : périmètres, compétences et actions, les notes territoriales de l'ADCF, décembre 2009).

${ }^{16}$ Chapitre II du Titre III « Développement et simplification de l'intercommunalité », articles 35 à 61.

${ }^{17}$ Au lieu du $1^{\text {er }}$ janvier 2014, mais les députés et les sénateurs, sur les conseils des associations d'élus locaux, ont cru bon de raccourcir les délais afin de laisser plus de temps entre la stabilisation des périmètres et les prochaines élections municipales-communautaires qui auront lieu en mars 2014.

18 Décret $n^{\circ} 2011-122$ du 28 janvier 2011 sur l'organisation et le fonctionnement des commissions départementales de coopération intercommunale. L'article 53 modifiant l'article L. 5211-43 du CGCT précise que les $\mathrm{CDCl}$ seront dorénavant composées de $40 \%$ de représentants de communes, $40 \%$ de représentants $\mathrm{d}^{\prime} \mathrm{EPCl}$ à $\mathrm{FP}$ et de $5 \%$ pour les représentants des syndicats mixtes et des syndicats de communes.
} 
Le schéma a notamment pour objet de prévoir les modalités concrètes de la couverture intégrale du territoire national par l'intercommunalité ${ }^{19}$, ainsi que les modalités de rationalisation des périmètres des intercommunalités existantes, en application d'un certain nombre d'orientations:

- Seuil démographique de 5000 habitants (sauf en zone de montagne) ${ }^{20}$;

- Amélioration de la cohérence spatiale en fonction du périmètre des unités urbaines, des bassins de vie et des SCOT ;

- Renforcement des solidarités financières ${ }^{21}$;

- Réduction du nombre des syndicats et le transfert éventuel de leurs compétences à des EPCl à FP;

- Rationalisation des structures compétentes en matière d'aménagement de l'espace, de protection de l'environnement et de respect des principes de développements durable.

En application de ces orientations le schéma peut donc proposer la création, la transformation, la fusion ainsi que la modification des périmètres des $\mathrm{EPCl}$ à $\mathrm{FP}$, ainsi que la suppression, la transformation et la fusion de syndicats de communes et de syndicats mixtes.

Au cours de cette année 2011, le projet de schéma ainsi élaboré par le préfet est adressé pour avis aux conseils municipaux des communes et aux organes délibérants des $\mathrm{EPCl}$ concernés et le tout (projet + avis) est ensuite transmis à la $\mathrm{CDCl}$ qui peut y intégrer des modifications adoptées à la majorité des $2 / 3$ de ses membres en conformité avec les orientations de la loi.

Le schéma est enfin arrêté et publié au plus tard le 31 décembre 2011 et durant l'année 2012, pour mettre en œuvre ses prescriptions, le préfet devra solliciter l'accord des conseils municipaux ${ }^{22}$. Après quoi, dans les 5 premiers mois de 2013, il pourra mettre en œuvre les propositions du schéma refusées précédemment par les communes, sous le contrôle de la $\mathrm{CDCl}$ qui pourra, à la majorité qualifiée des $2 / 3$ de ses membres, amender les projets du préfet.

Par ailleurs, au-delà même du $1^{\text {er }}$ juin 2013, l'article 38 de la loi prévoit que le préfet pourra rattacher une commune isolée ou "créant une enclave ou une discontinuité au sein d'une intercommunalité à un EPCI à $F P$ ", et passer outre le refus du conseil communautaire d'intégrer la commune, sauf si la $\mathrm{CDCl}$ s'est prononcée à la majorité des $2 / 3$ de ses membres en faveur d'un autre projet de rattachement que le préfet devra dès lors mettre en œuvre. Cet aspect de la réforme est tout à fait novateur puisque jusqu'à présent, il n'y avait aucun moyen juridique d'intégrer des communes qui s'y opposaient formellement.

\footnotetext{
${ }^{19}$ Sauf dans les départements de Paris, des Hauts-de-Seine, de Seine Saint Denis et du Val de Marne.

${ }^{20}$ Ce seuil peut néanmoins être abaissé par le préfet pour tenir compte des caractéristiques géographiques particulières de certains espaces; on pense notamment aux espaces ruraux les moins denses.

${ }^{21}$ Pour éviter les fameux « clubs de riches » et les intercommunalités « défensives ".

${ }^{22}$ Exprimé à la majorité de $50 \%$ des communes représentant $50 \%$ de la population totale avec un pouvoir de blocage de la commune représentant au moins le $1 / 3$ de la population totale ; à ce stade de la procédure seul I'avis des organes délibérants des EPCl est requis.
} 
Ensuite, L'article 42 prévoit la modification de la procédure de fusion des communautés ${ }^{23}$ qui semblait jusqu'à présent trop contraignante au gouvernement pour susciter suffisamment de fusions ${ }^{24}$.

Enfin, les articles 44 à 50 de la loi du 16 décembre 2010 ont pour objectif de favoriser la réduction du nombre de syndicats, conformément aux prescriptions du schéma ${ }^{25}$ et l'article 51 abroge l'article 22 de la loi du 4 février 1995 d'orientation pour l'aménagement et le développement du territoire qui prévoyait la création des pays. Cette disposition notamment a suscité une importante opposition, particulièrement en milieu rural.

On peut tout d'abord remarquer l'apparition de la notion de bassin de vie dans les différentes orientations devant guider la formation des nouveaux périmètres intercommunaux; c'est en effet la première fois qu'un territoire fonctionnel est pris en considération pour déterminer un territoire institutionnel, si l'on exclue la vague notion d'agglomération qui avait fait son apparition dans la loi Chevènement de 1999.

Par ailleurs, si certains élus, notamment les élus ruraux, ont dénoncé la « caporalisation » ou la « marche forcée » de l'intercommunalité, la plupart se sont félicités qu'il y ait de nouveau « un pilote dans l'avion » de l'intercommunalité et que l'Etat assume enfin ce rôle.

Ce qui est sûr, c'est que si l'on souhaite l'achèvement de la carte intercommunale, avec en tête l'idée d'un maillage intercommunal qui constitue le prolongement (voire la substitution) du maillage communal, on ne peut qu'en appeler à un certain retour de l'autoritarisme en la matière, certaines résistances à l'intercommunalisation sont trop profondément ancrées et bloquent durablement les situations. La liberté a jusqu'à présent suscité en effet pas mal $d^{\prime}$ 'inertie en la matière.

Tout dépendra finalement de la façon dont les préfets et les élus locaux vont mener l'affaire. Si les uns et les autres font un bon usage de la concertation, si le préfet amorce le dialogue dans un esprit d'ouverture et de discussion et que les élus ne pratiquent pas la politique de la chaise vide notamment au sein des $\mathrm{CDCl}$ et s'investissent dans la concertation, on pourra sans doute obtenir de bons résultats qui satisferont tout le monde.

\footnotetext{
${ }^{23}$ Il est prévu une consultation obligatoire de la $\mathrm{CDCl}$ dans tous les cas avec possibilité de modifier le périmètre de la fusion à la majorité des $2 / 3$ de ses membres ainsi qu'une possibilité d'initiative du projet de fusion et l'intégration de communes membres d'EPCI à FP « lorsque leur inclusion est de nature à assurer la cohérence spatiale et économique ainsi que la solidarité financière nécessaires au développement du nouvel EPCI dans le respect du SDCl». L'avis des conseils communautaires des EPCl susceptibles de fusionner est simplement requis au lieu de leur accord et, en contrepartie, la condition de majorité des conseils municipaux est renforcée : la règle de l'accord de la majorité qualifiée des $2 / 3$ des conseils municipaux des communes représentant la moitié de la population totale (ou l'inverse) à l'échelle du périmètre du futur EPCI est maintenue, mais y est ajouté l'accord d'au moins un tiers des communes de chacun des EPCI concernés. Enfin, les communes se prononceront en même temps sur le nouveau périmètre et sur le statut du nouvel $\mathrm{EPCl}$ et les compétences subordonnées à la reconnaissance de leur intérêt communautaire ne seront transférées au nouvel EPCl qu'une fois que celui-ci a été défini dans un délai de deux ans à compter de l'arrêté prononçant la fusion ; jusqu'à là, l'ancien intérêt communautaire est maintenu dans les anciens périmètres correspondant à chacun des EPCI fusionnés.

${ }^{24}$ En effet, il n'y a eu que 5 fusions en 2005, 4 en 2006, 9 en 2007, 10 en 2008 et 13 en 2009.

${ }^{25} \mathrm{Au} 1^{\text {er }}$ janvier 2010, la DGCL dénombrait encore 12184 syndicats de communes, dont $88 \%$ de SIVU (10 789) auxquels il convient d'ajouter 3194 syndicats mixtes, chiffre en constante augmentation, puisqu'ils n'étaient que 1454 en 1999, 2687 en 2006 et 3064 en 2009. Par ailleurs, une commune adhère d'ailleurs en moyenne à plus de 4 syndicats et 5 départements en comptent plus de 250 .
} 
Cependant, deux difficultés de taille se profilent en ce qui concerne la rationalisation des périmètres. La première a trait aux nécessités de recompositions territoriales liées à l'objectif de solidarité financière qui reste très flou en raison des incertitudes liées aux nouvelles réformes fiscales qui touchent de plein fouet les intercommunalités et qui vont profondément modifier les rapports de force au sein et entre les intercommunalités. La seconde concerne les incitations en faveur des mutualisations de services qui vont nécessairement limiter la taille des périmètres intercommunaux. Ce qui revient finalement à dire qu'il n'y a pas de périmètre pertinent en soi, il n'y a que des périmètres pertinents par rapport aux compétences exercées.

\section{La gouvernance}

L'article 8- $-1^{\circ}$, alinéa 1 de la loi du 16 décembre 2010 dispose : "Les métropoles, CU, CA et CC sont administrées par un organe délibérant composé des délégués des communes membres élus dans le cadre de l'élection municipale au suffrage universel direct pour toutes les communes dont le conseil municipal est élu au scrutin de liste ${ }^{26}$, dans les conditions fixées par la loi. Les autres EPCI sont administrés par un organe délibérant composé de délégués élus par les conseils municipaux des communes membres dans les conditions fixées à l'article L. $2122-7^{27}$ ».

Cette disposition figurait déjà dans le rapport Mauroy de $2000^{28}$. Depuis, l'élection des conseillers communautaires fait partie des engagements de toutes les formations politiques, et revient dans les propositions de toutes les commissions et missions qui planchent sur la question de l'organisation territoriale en France $^{29}$, sans que jamais une loi n'ait jusqu'à présent permis d'engager une telle réforme ${ }^{30}$.

\section{a. La nécessité d'une réforme}

L'intercommunalité doit être un lieu de démocratie d'abord parce qu'elle est un lieu de pouvoir, ensuite parce qu'elle est un véritable territoire et enfin parce qu'elle doit des comptes à sa population.

La plupart des décisions intéressant l'action publique locale sont en en effet transférées aux instances intercommunales, les communes ne conservant plus qu'un pouvoir subalterne en matière de gestion de proximité ; dès lors il n'y a pas de sens à ne maintenir la démocratie que là où le pouvoir local se vide petit à petit de son contenu et à refuser de la mettre en œuvre là où il s'est finalement déplacé.

\footnotetext{
${ }^{26}$ C'est-à-dire les communes de plus de 500 habitants.

${ }^{27}$ C'est-à-dire les syndicats.

${ }^{28}$ Commission pour l'avenir de la décentralisation, Refonder l'action publique locale, La Documentation Française, 2000.

${ }^{29} C^{\prime}$ est donc notamment le cas de la préconisation $n^{\circ} 7$ du rapport d'étape de la mission Belot et de la proposition $\mathrm{n}^{\circ} 7$ du rapport Balladur.

${ }^{30} \mathrm{~V}$. notre article, L'élection des conseillers communautaires au suffrage universel direct : l'intercommunalité entre fiction et réalité, in Collectivités Territoriales, Intercommunalité, Jurisclasseur, janvier 2006, p. 4 et suiv.
} 
Les transferts de compétences des communes vers les intercommunalités entraînent d'ailleurs des transferts de contrats, d'équipements, de services et de personnels inhérents aux compétences transférées, ce qui sera d'autant plus visible sous l'effet de la mutualisation des services prévue dans la réforme actuelle.

Les budgets intercommunaux sont particulièrement importants en volumes, les enjeux financiers se situent à l'heure actuelle donc largement au niveau intercommunal.

Les EPCI bénéficient en outre d'une dynamique institutionnelle inédite, puisqu'on le sait, au $1^{\text {er }}$ juin 2013, toutes les communes françaises devront être intercommunalisées.

Enfin, l'article 14 de la Déclaration des droits de l'homme et du citoyen du 26 août 1789 pose le principe selon lequel " tous les citoyens ont le droit de constater par eux-mêmes ou par leurs représentants, la nécessité de la contribution publique, de la consentir librement, d'en suivre l'emploi, et d'en déterminer la quotité, l'assiette, le recouvrement et la durée ". Or, le fait que les structures intercommunales lèvent l'impôt entraînent nécessairement un lien de représentation démocratique entre les citoyens et les élus et ce lien est d'ailleurs d'autant plus affirmé qu'aujourd'hui, en raison de la réforme de la TP, les intercommunalités lèvent principalement les « impôts ménages ». Un nouveau rapport s'établit désormais entre la communauté et les habitants, usagers et dorénavant contribuables de cette communauté et l'exigence de transparence et de lisibilité des choix opérés par l'intercommunalité en sera d'autant plus accrue et doit immanquablement faire apparaître un véritable "citoyen intercommunal $"$.

II n'est donc plus possible de passer encore sous silence "le fait intercommunal " et les enjeux qu'il soulève, comme cela a été encore trop souvent le cas lors des élections municipales de 2008 et seule la mise en place d'un scrutin direct permettra aux électeurs d'avoir connaissance de ces enjeux. On compte d'ailleurs sur la représentation accrue des oppositions municipales au sein des assemblées communautaires, désormais permise par les dispositions de la réforme, pour médiatiser les enjeux communautaires, même si cette représentation entraîne le risque d'une politisation des débats communautaires au détriment du sacro-saint "consensus " qui semble avoir été la marque intercommunale depuis si longtemps. II est désormais nécessaire que les questions intercommunales ne soient plus une affaire de spécialistes réglées dans "l'entre soi " d'une arène communautaire fermée aux citoyens ${ }^{31}$.

\section{b. Les dispositions de la loi du $\mathbf{1 6}$ décembre $\mathbf{2 0 1 0}$}

L'article 8 a donc retenu le système de scrutin dit de " fléchage " pour les communes de plus de 500 habitants, déjà préconisé dans le rapport Mauroy de 2000, sur le modèle des élections municipales et d'arrondissement de Paris, Lyon, Marseille: les candidats au mandat de conseiller municipal et aux fonctions de délégué communautaire figureront sur une seule et même liste, les premiers de la liste ayant vocation à siéger au conseil municipal

\footnotetext{
${ }^{31} \mathrm{~V}$. notre article, L'élection des conseillers communautaires au suffrage universel direct : l'intercommunalité entre fiction et réalité, prec.
} 
de leur commune et au conseil communautaire, les suivants sur la liste ne siégeant qu'au conseil municipal de leur commune.

Dans les communes de moins de 500 habitants, les délégués des communes sont le maire et les conseillers municipaux désignés dans l'ordre du tableau établi lors de l'élection municipale.

Un certain nombre d'observateurs ou d'élus locaux se plaignent cependant que le système proposé par la réforme n'est pas encore satisfaisant.

Certains auraient souhaité un mode de scrutin totalement autonome par rapport aux élections municipales, organisé dans le cadre d'une circonscription intercommunale, mais ce type de scrutin aurait signé de façon radicale le divorce entre communes et intercommunalité puisque il y aurait eu nécessairement dissociation entre les élus municipaux et les élus communautaires. Or, l'objectif est bien ici à court terme en tous cas de renforcer le couple commune-intercommunalité et pas de le dissoudre.

D'autres encore postulaient pour que seuls les présidents de communauté, voire seulement les présidents de CU soient élus au suffrage universel direct, mais ce système ne s'accorde absolument pas avec notre culture électorale et aurait accentué la présidentialisation de l'intercommunalité au détriment des voix des communes, notamment des plus petites d'entre elles.

Par ailleurs, pour tirer toutes les conséquences de l'élection au suffrage universel direct des délégués communautaires, l'article 9 est censé améliorer l'équilibre de la représentation des communes membres au sein du conseil communautaire en prenant davantage en compte leur poids démographique.

Cependant, là où le projet de loi initial prévoyait d'imposer la composition des conseils communautaires et la répartition des sièges, selon un tableau tenant compte du poids démographique de la communauté, le nouveau texte assouplit le système en permettant que, dans les CC et les CA, les communes décident, comme auparavant, par accord à la majorité qualifiée de leurs conseils municipaux de la composition et de la répartition des sièges. Cette répartition à l'amiable devra cependant tenir compte de la population de chaque commune, permettre que chacune dispose d'au moins un siège, interdire à une commune de disposer de plus de la moitié des sièges, et enfin ne pas prévoir un nombre total de sièges supérieur à $10 \%$ de celui prévus dans le tableau.

Précisément, dans les $\mathrm{CU}$ et les métropoles et à défaut d'accord à la majorité qualifiée dans les autres communautés, le nombre de délégués des communes est fonction de la population totale de la communauté, les sièges étant répartis à la représentation proportionnelle en fonction du poids démographique de la population des communes, en fonction du tableau ci-après : 


\begin{tabular}{|l|c|}
\hline \multicolumn{1}{|c|}{ Population municipale de la communauté } & Nombre de sieges \\
\hline De moins de 3500 habitants & 16 \\
\hline De 3500 à 4999 habitants & 22 \\
\hline De 5000 à 9999 habitants & 26 \\
\hline De 10000 à 19999 habitants & 30 \\
\hline De 20000 à 29999 habitants & 34 \\
\hline De 30000 à 39999 habitants & 38 \\
\hline De 40000 a 49999 habitants & 40 \\
\hline De 50000 à 74999 habitants & 42 \\
\hline De 75000 à 99999 habitants & 48 \\
\hline De 100000 à 149999 habitants & 56 \\
\hline De 150000 à 199999 habitants & 64 \\
\hline De 200000 à 249999 habitants & 72 \\
\hline De 250000 à 349999 habitants & 80 \\
\hline De 350000 à 499999 habitants & \\
\hline De 500000 à 699999 habitants & \\
\hline De 700000 à 1000 000 habitants & \\
\hline Plus de I 000000 habitants & \\
\hline
\end{tabular}

En outre, les communes peuvent décider à la majorité qualifiée de créer et répartir un nombre de sièges supplémentaires inférieur ou égal à $10 \%$ du nombre de sièges issu de l'application du tableau, et pour les CU et les métropoles, cela peut avoir pour conséquence qu'une commune détienne plus de la moitié des sièges.

Enfin, cet article 9 prévoit également de limiter le nombre de vice-présidents des $\mathrm{EPCl}$ à $20 \%$ de l'effectif de l'assemblée délibérante.

Les dispositions de l'article 9 sont d'application immédiate pour les nouveaux $E P C l$ créés après la publication de la loi ou dont les périmètres auront été modifiés mais ne s'appliquera aux autres qu'à compter du prochain renouvellement issu des élections municipales de 2014.

\section{Les moyens}

\section{a. Le renforcement des moyens techniques et administratifs}

En premier lieu, les articles 65 et 66 de la loi du 16 décembre 2010 ont pour objet de renforcer les procédures de mutualisation des services au sein du bloc local, entre communes et communautés et de les sécuriser au regard des règles de droit communautaire relatives au droit de la concurrence, dans un objectif de rationalisation des coûts en termes d'agents publics et donc d'économie d'échelle.

Ainsi, en ce qui concerne les compétences transférées à l'intercommunalité, l'article L.52114-1 du CGCT relatif à la mise à disposition de services entre communes et intercommunalité (mutualisation ascendante ou descendante) est modifié de façon à ce que les modalités de la 
mise à disposition ainsi que les conditions de leur remboursement financier soient prévues dans le cadre d'une convention.

Par ailleurs, indépendamment des transferts de compétences, les communautés peuvent créer des services fonctionnels communs composés d'agents de la communauté et/ou d'agents communaux mis à disposition de plein droit. Cette mise à disposition est réglée par convention et en fonction de la mission, les agents seront placés sous l'autorité fonctionnelle du maire ou du président de la communauté. Les frais de remboursement pourront être imputés éventuellement sur l'attribution de compensation.

Ensuite, l'article 66 permet à une communauté de se doter de biens mis à disposition des communes membres, les modalités de mise à disposition étant prévues par un règlement.

Enfin, l'article 67 prévoit la création en début de mandat dans chaque communauté d'un schéma de mutualisation des services à mettre en œuvre pendant la durée du mandat et, chaque année, l'avancement du schéma de mutualisation fera l'objet d'une communication du président à l'organe délibérant. II s'agit ici d'une obligation de moyen et non d'une obligation de résultat, mais ce rendez-vous annuel est incontestablement un progrès au regard des réticences antérieures que le législateur avait manifesté à l'égard de la mutualisation des services avant 2004.

En second lieu, l'article 63 prévoit que lorsque l'EPCI détient les compétences " assainissement ", " élimination des déchets ménagers ", " accueil et habitat des gens du voyage ", son président se voit attribuer automatiquement les pouvoirs de police spéciale afférents à ces compétences. En revanche, en ce qui concerne la compétence "voirie ", le transfert des pouvoirs de police spéciale en matière de stationnement et de circulation ne sera pas automatique.

\section{b. Le renforcement des solidarités financières et fiscales}

Les articles 70 et 72 prévoient qu'il peut être décidé à l'unanimité des conseils municipaux d'unifier la DGF à l'échelle intercommunale ${ }^{32}$ ainsi que tout ou partie de la fiscalité directe sur les ménages ${ }^{33}$.

Mais c'est sur la question de la refonte totale du panier de ressources des communautés, liée au transfert des taxes ménages départementales vers l'intercommunalité et à la baisse de rendement de l'impôt économique, à la suite de la suppression de la TP, que portent les principales inquiétudes des élus locaux qui craignent l'impact de la réforme fiscale et le changement de règles en milieu de partie. Les nouvelles recettes qui viennent se substituer à

\footnotetext{
${ }^{32}$ Dans ce cas, la communauté met en place une dotation de reversement dont le montant versé à chaque commune est fixé par le conseil à la majorité des $2 / 3$ des suffrages exprimés en tenant compte prioritairement, comme pour la dotation de solidarité communautaire, de l'écart entre le revenu par habitant de la commune et le revenu moyen par habitant de l'EPCl et de l'insuffisance de potentiel fiscal par habitant de la commune au regard du potentiel fiscal communal moyen par habitant sur le territoire de I'EPCI, mais contrairement à la dotation de solidarité communautaire, il s'agira d'une dépense obligatoire.

${ }^{33}$ Dans ce cas, pour chaque taxe dont l'unification est décidée, le taux est voté par le conseil communautaire dans les mêmes conditions que celles applicables aux communes, et l'on fonctionne selon le même système appliqué antérieurement pour la TPU (taux moyen pondéré à ne pas dépasser la 1 ère année, période de lissage des taux sur 10 ans ... )
} 
la TPU pénalisent en effet les territoires qui avaient misé sur l'industrie et favorisent les territoires de type résidentiel, alors que jusqu'à présent les intercommunalités avaient été incitées à privilégier la compétence de développement économique. On assiste donc à la fin du modèle de spécialisation fiscale et au passage obligé à la fiscalité mixte et ce, sans qu'aucune d'entre elles n'aient pu anticiper une telle mutation de leurs politiques de développement et cette modification profonde de la géographie fiscale des intercommunalités.

En outre, un contexte financier très contraint vient également peser sur elles et le gel annoncé des dotations de l'Etat, la fin des financements croisés, les nouvelles orientations prévues pour la DGF, la suppression de la clause générale de compétence des départements et des régions, le recul de l'Etat territorial ainsi que le déclin des services publics sont autant d'inconnus qui incitent les intercommunalités à un immobilisme forcé.

\section{Les nouvelles formes de coopération}

Très paradoxalement, alors qu'il était semble-t-il urgent d'alléger le millefeuille institutionnel français qui était jugé trop indigeste, de nouveaux territoires ont cependant fait leur apparition, ajoutant ainsi des couches supplémentaires : il s'agit des métropoles, des pôles métropolitains et des communes nouvelles.

\section{a. Les métropoles}

La Métropole correspond au souci d'adapter les structures administratives à la diversité des territoires, de prendre en considération la montée en puissance du fait urbain et de mieux insérer les agglomérations françaises dans la compétition entre les grandes agglomérations européennes et internationales.

Cependant, si en juillet 2009, dans l'avant-projet de loi, la métropole était une collectivité territoriale de plein exercice dotée des compétences d'une $\mathrm{CU}$ et de l'ensemble des compétences départementales sur son périmètre ${ }^{34}$, dans le projet de loi déposé en octobre 2009 et finalement promulgué en décembre 2010, si le label «métropole » demeure, le statut quant à lui est pratiquement calqué sur celui de l'actuelle $\mathrm{CU}$ et fait perdre à la métropole à la fois son intérêt et son caractère novateur.

L'article 12 de la loi prévoit en effet que la métropole est un nouvel EPCl, regroupant sur la base du volontariat, plusieurs communes qui forment un ensemble de plus de 500000 habitants d'un seul tenant et sans enclave, constitué pour produire un projet d'aménagement et de développement économique, écologique, éducatif, culturel et social de son territoire, afin d'en améliorer la compétitivité et la cohésion.

\footnotetext{
${ }^{34}$ Selon les vœux du Comité Balladur, l'avant-projet de loi consacrait la métropole en tant que collectivité territoriale, puisqu' "II [allait] de soi dans l'esprit du comité que ces onze métropoles auraient vocation à constituer une manière d'avant-garde de l'intégration progressive des communes dans des ensembles plus vastes et dotés de la qualité de collectivité locale et non plus seulement d'Etablissement Public " (Rapport du Comité pour la réforme des collectivités locales, prec., p. 88).
} 
La création de la métropole nécessite l'accord des communes aux conditions de majorité qualifiée classiquement retenues en matière d'intercommunalité ${ }^{35}$. Elle est en revanche imposée aux départements et aux régions concernées, puisque seul leur avis est requis. Elle peut aussi être le fait de la transformation d'un EPCI à FP, après délibérations concordantes du conseil communautaire et des conseils municipaux des communes, aux conditions de majorité qualifiée classiques.

Les sénateurs ayant contribué au fil des lectures à détricoter le statut de la métropole et à vider de leur substance ses dernières spécificités, le champ d'intervention de la métropole est finalement fort peu élargi par rapport à celui de la $\mathrm{CU}$, puisqu'elle ne reçoit plus que les attributions départementales en matière de transports scolaires, de gestion des voies départementales et de zones d'activités et les compétences départementales et régionales en matière de promotion à l'étranger du territoire et de ses activités économiques.

A cela s'ajoutent, mais seulement à titre facultatif, c'est-à-dire avec l'accord du département ou de la région, la compétence en matière de collèges, tout ou partie des compétences départementales en matière d'action sociale, de développement économique, de tourisme, et en matière culturelle et de construction, d'exploitation et d'entretien des équipements et infrastructures destinés à la pratique du sport, ainsi que la compétence lycées et tout ou partie des compétences régionales en matière de développement économique.

En outre, si la métropole en fait la demande, l'Etat pourra décider de lui transférer des grands équipements ou infrastructures situés sur son territoire.

Enfin, du point de vue financier et fiscal, là encore cette métropole n'aura finalement pas réussi à franchir le saut qualitatif que souhaitait le gouvernement, puisque rien ne la distingue des autres communautés, si bien que désormais, la métropole n'apparaît plus que comme une "énième " intercommunalité, une "CU bis", déstructurant l'architecture pyramidale mise en place par la loi Chevènement de 1999. Pour la restaurer, il a d'ailleurs fallu rabaisser le seuil démographique de la CU à 450000 habitants $^{36}$.

\section{b. Les pôles métropolitains}

L'article 20 de la loi prévoit que le pôle métropolitain est constitué sous la forme d'un syndicat mixte qui regroupe des EPCl à FP formant un ensemble de plus de 300000 habitants ${ }^{37}$ dont un des $\mathrm{EPCl}$ au moins comporte plus de 150000 habitants $^{38}$ sans exigence de continuité spatiale.

\footnotetext{
${ }^{35}$ Ainsi, la métropole peut se constituer par regroupement de commune, à l'initiative d'une ou plusieurs d'entre elles, avec accord des conseils municipaux des communes concernées à la majorité qualifiée classique pour la création des $\mathrm{EPCl}$ ( $2 / 3$ des conseils municipaux représentant plus de la $1 / 2$ de la population totale ou l'inverse).

${ }^{36}$ D'ailleurs, dans le même état d'esprit, l'article 19 dispose que "Le seuil démographique de 50000 habitants [de création d'une CA] est réduit à 30000 habitants lorsque la communauté d'agglomération comprend le cheflieu du département. Le seuil démographique de 50000 habitants peut également être apprécié en prenant en compte la population telle que définie à l'article L. 2334-2, à la double condition que cette dernière excède ce seuil d'au moins $20 \%$ et qu'elle excède la population totale de plus de $50 \%$. ". Il s'agit en effet de prendre en compte les variations de population saisonnière dans les territoires à forte population touristique.

${ }^{37} 450000$ habitants prévus dans le projet de loi.
} 
Il s'agit donc d'un outil de coopération souple entre EPCI, apparaissent à la fois comme un lot de consolation destiné à la quinzaine d'intercommunalités comprenant entre 150000 et 500000 habitants et exclues de fait du club des métropoles, et à la fois comme un dispositif qui complexifie encore plus la réforme territoriale.

L'absence de continuité spatiale est évidemment, dans bien des cas, la condition sine qua non de la réalisation d'un tel ensemble, mais paraît contradictoire avec les exigences affichées par le gouvernement, notamment en ce qui concerne l'achèvement et la rationalisation de la carte intercommunale.

Cependant, la souplesse de cet instrument permettra peut-être de traiter plus efficacement les questions interterritoriales que les intercommunalités ne peuvent pas appréhender.

\section{c. Les communes nouvelles}

L'article 21 dispose qu' " une commune nouvelle peut être créée en lieu et place de communes contiguës». Ce nouveau dispositif vient se substituer à l'ancien dispositif de fusion de communes issu de la Loi Marcellin de $1971^{39}$, dont on a sait qu'il s'était révélé peu efficace.

Il ne s'agit donc ni plus ni moins d'une énième tentative de fusion de communes, guère audacieuse qui plus est, alors que les impératifs de réduction du nombre de communes auraient sans doute exigé une mesure plus radicale. La commission Balladur avait à cet égard préconisé que ce nouveau dispositif permette à la fois de réduire de façon significative le nombre excessif de nos communes et de transformer des intercommunalités en communes de plein exercice. Le mode de création prévu initialement passait par un vote à la majorité des conseils municipaux et un référendum de la population et une bonification de la DGF était prévu à titre incitatif. Or, il ne reste ici rien de ce dispositif ${ }^{40}$ et certains ont cru bon

\footnotetext{
${ }^{38} 200000$ habitants prévus dans le projet de loi.

${ }^{39}$ Loi $n^{\circ} 71-588$ du 16 juillet 1971 sur les fusions et regroupements de communes.

${ }^{40}$ La création de cette commune nouvelle est soumise soit à la demande de tous les conseils municipaux (donc ici unanimité), soit à la demande de la majorité qualifiée des conseils municipaux des communes membres d'un $\mathrm{EPCl}$ à $\mathrm{FP}$ (2/3 au moins des conseils municipaux représentant plus des $2 / 3$ de la population), soit à la demande de l'organe délibérant de l'EPCI à FP en vue de la création d'une commune nouvelle en lieu et place de toutes ses communes membres (Plus des $2 / 3$ des conseils municipaux représentant + des $2 / 3$ de la population), soit à la demande du préfet.

Dans les deux derniers cas, il faudra en outre réunir l'accord des conseils municipaux des communes membres ou des communes concernées aux même conditions de majorité qualifiée que dans le second cas.

Lorsque la demande ne fait pas l'objet de l'unanimité des conseils municipaux de toute les communes concernées, mais est formée dans les conditions de majorité qualifiée susmentionnées, on organisera également un référendum local. La création ne pourra dès lors être décidée par le préfet que si ce référendum recueille la majorité absolue des suffrages dans chaque commune et satisfait à une condition de quorum fixée à plus du quart des électeurs inscrits et que la participation totale au scrutin est supérieure à la moitié des électeurs inscrits dans l'ensemble du périmètre.

En outre, si en principe la commune nouvelle se substitue de plein droit aux anciennes communes fusionnées, et, sauf délibération contraire du conseil municipal de la commune nouvelle, ces dernières peuvent cependant devenir des communes déléguées dont le maire et le conseil disposeraient des mêmes attributions que le maire et le conseil d'arrondissement dans le système PLM issu de la loi du 31 décembre 1982.
} 
d'ironiser en espérant être invités à l'inauguration de la première commune nouvelle, prédisant qu'il n'y en aurait pas de deuxième ${ }^{41}$.

Et pourtant, rappelons tout de même que La France compte 36793 communes, ce qui représente $40 \%$ des communes de l'Union, qu'environ une commune sur deux a moins de 400 habitants et une sur quatre en a moins de 200. Rappelons enfin que la commune moyenne française " pèse " à peine 1720 habitants, lorsque la moyenne européenne est de 5000 habitants et qu'au Danemark une commune moyenne compte 55580 habitants, pour 36000 habitants aux Pays-Bas, 31000 en Suède, 17900 en Belgique et 5900 en Allemagne.

Cette situation française tout à fait exceptionnelle aurait sans doute nécessité que l'on se penche sur cette question de fusion de communes de façon plus efficiente et non que l'on reproduise quasiment à l'identique un processus de fusion qui a fait les preuves de son inefficacité! C'était un des enjeux de la réforme à côté duquel elle est complètement passée.

\section{Cécile Jebeili}

Maître de conférences en droit public

Département de Géographie-Aménagement Université Toulouse le Mirail UMR Dynamiques rurales

\footnotetext{
${ }^{41}$ Jean-Patrick Courtois, rapporteur UMP au Sénat de la loi du 16 décembre 2010.
} 